\title{
Fast Healthcare Interoperability Resources, Clinical Quality Language, and Systematized Nomenclature of Medicine-Clinical Terms in Representing Clinical Evidence Logic Statements for the Use of Imaging Procedures: Descriptive Study
}

Eseosa Odigie $^{1^{*}}$, BSc; Ronilda Lacson ${ }^{1,2^{*}}, \mathrm{MD}, \mathrm{PhD}$; Ali Raja ${ }^{1,2}$, MBA, MD; David Osterbur ${ }^{1,3}, \mathrm{PhD}$; Ivan Ip ${ }^{1,2}, \mathrm{MPH}$, MD; Louise Schneider ${ }^{1,2}$, MD; Ramin Khorasani ${ }^{1,2}$, MPH, MD

\footnotetext{
${ }^{1}$ Center for Evidence-Based Imaging, Brigham and Women's Hospital, Brookline, MA, United States

${ }^{2}$ Harvard Medical School, Boston, MA, United States

${ }^{3}$ Countway Medical Library, Harvard Medical School, Boston, MA, United States

* these authors contributed equally
}

\section{Corresponding Author:}

Ronilda Lacson, MD, PhD

Center for Evidence-Based Imaging

Brigham and Women's Hospital

20 Kent Street

Brookline, MA, 02445

United States

Phone: 16175259712

Fax: 16175257575

Email: rlacson@rics.bwh.harvard.edu

\begin{abstract}
Background: Evidence-based guidelines and recommendations can be transformed into "If-Then" Clinical Evidence Logic Statements (CELS). Imaging-related CELS were represented in standardized formats in the Harvard Medical School Library of Evidence (HLE).

Objective: We aimed to (1) describe the representation of CELS using established Systematized Nomenclature of Medicine-Clinical Terms (SNOMED CT), Clinical Quality Language (CQL), and Fast Healthcare Interoperability Resources (FHIR) standards and (2) assess the limitations of using these standards to represent imaging-related CELS.

Methods: This study was exempt from review by the Institutional Review Board as it involved no human subjects. Imaging-related clinical recommendations were extracted from evidence sources and translated into CELS. The clinical terminologies of CELS were represented using SNOMED CT and the condition-action logic was represented in CQL and FHIR. Numbers of fully and partially represented CELS were tallied.

Results: A total of 765 CELS were represented in the HLE as of December 2018. We were able to fully represent 137 of 765 $(17.9 \%)$ CELS using SNOMED CT, CQL, and FHIR. We were able to represent terms using SNOMED CT in the temporal component for action ("Then") statements in CQL and FHIR in 755 of 765 (98.7\%) CELS.

Conclusions: CELS were represented as shareable clinical decision support (CDS) knowledge artifacts using existing standards-SNOMED CT, FHIR, and CQL_to promote and accelerate adoption of evidence-based practice. Limitations to standardization persist, which could be minimized with an add-on set of standard terms and value sets and by adding time frames to the CQL framework.
\end{abstract}

(JMIR Med Inform 2019;7(2):e13590) doi: 10.2196/13590

\section{KEYWORDS}

knowledge representation; guidelines; evidence-based medicine; clinical decision support 


\section{Introduction}

\section{Background}

Imaging clinical decision support (CDS) applies health information technology (IT) to inform clinical decision making at the point of care regarding the need for imaging or the optimal study based on the best available evidence [1]. Legislation has called for the use of health IT, including CDS, for health promotion and health quality improvement $[2,3]$. Subsequently, regulations promulgated in response to the Protecting Access to Medicare Act (PAMA) state that health care providers should reference appropriate use criteria or evidence-based clinical knowledge while ordering certain advanced imaging exams [4]. Such an evidence-based approach to appropriate medical imaging by way of CDS systems can help mitigate health care costs and imaging utilization, while providing appropriate and safe health care to those who require these procedures [5-7].

Many guidelines, recommendations, systematic reviews, and clinical decision rules have been published or endorsed by national societies in the peer-reviewed literature and as best practices by other provider groups related to appropriate use of advanced imaging procedures for certain indications. The knowledge contained in these recommendations and guidelines can be transformed into Clinical Evidence Logic Statements (CELS) that can be implemented into CDS systems. However, to be widely shared and usable in such systems, CELS must be translated into established standardized syntax and formats such as Systematized Nomenclature of Medicine-Clinical Terms (SNOMED CT) [8], Clinical Quality Language (CQL) [9], and Fast Healthcare Interoperability Resources (FHIR) [10]. SNOMED International does not charge for the use of SNOMED CT in SNOMED International Member countries or territories; CQL and FHIR are Health Level Seven (HL7) standards and are available at no cost under a licensing agreement by which HL7 will retain its copyright. Key components of each standard are summarized in the subsections that follow.

\section{Systematized Nomenclature of Medicine-Clinical Terms Compositional Grammar}

SNOMED CT compositional grammar is a standard ontology for representing clinical concepts and establishes relationships between them [8]. Clinical terms such as "X-ray knee" can be modeled in SNOMED CT, where each concept is linked to an identifying number. Concepts in SNOMED CT are organized into expressions. Precoordinated expressions are represented by a single concept identifier. Postcoordinated expressions are those that are represented by combining two or more concept identifiers. SNOMED CT establishes rules and hierarchies that define attributes, qualifiers, and relationships between concepts [11]. SNOMED CT also enables reference sets, which can be used to group SNOMED CT components (ie, concepts).

\section{Health Level Seven Clinical Quality Language Standard}

The HL7 CQL Specification was developed to standardize the representation of clinical logic for clinical quality improvement [12]. More specifically, CQL was developed with the target of harmonizing expression logic. An additional component of the
CQL Standard is the Expression Logical Model (ELM) [12]. Each CQL logic file is also represented as an ELM Extensible Markup Language (XML) document, which allows for an action to be represented for CDS. CQL files can reference clinical terms represented using SNOMED CT [8]. CQL files can also reference data models, such as the Quality Information and Clinical Knowledge (QUICK) logical model [13]. The QUICK data model defines the format and structure of the "retrieve" expressions in a CQL library. The retrieve declaration gathers a list of clinical data that is specific to the context of the patient or the population and to the retrieve itself.

\section{Fast Healthcare Interoperability Resources}

FHIR is a standard for sharing health care information with multiple functional areas known as resources [10]. These modules or individual components can be combined into a framework that can be implemented in a health care system. The modules are generated in a format that can be recognized and utilized by most health care systems, while also allowing for flexibility and customization of these resources through extensions. Data representation in FHIR can be in the XML, JavaScript Object Notation (JSON), or Turtle formats and it uses both CQL and SNOMED CT standards in its representations. The FHIR "decisionsupportrule" resource [14], expressed through the ELM, represents shareable knowledge artifacts for CDS.

The Harvard Medical School Library of Evidence (HLE) provides a repository of medical evidence, publicly available from the HLE website, from a range of recommendation sources that can be utilized in CDS systems $[15,16]$. Each unit of medical evidence is represented as a CELS of "If-Then" logic statement form (eg, If [age $>\mathrm{X}$ ] And [symptom] Then Not [procedure]). We aimed to (1) describe the representation of CELS using the established standards of SNOMED CT, CQL, and FHIR and (2) assess the limitations of using these standards to represent the CELS in the HLE.

\section{Methods}

\section{Study Design and Setting}

This descriptive study was exempt from the requirement of review from the Institutional Review Board as it did not include human subjects. The HLE currently contains imaging-related recommendations from clinical decision rules, professional society guidelines, and locally developed best practice guidelines [17]. As of December 20, 2018, there were a total of 765 completely graded CELS from 134 evidence sources in the HLE. A total of 235 of the CELS are Choosing Wisely content [18], pertaining to Priority Clinical Areas (eg, cervical or neck pain and suspected pulmonary embolism) specified by the Centers for Medicare and Medicaid Services [4,19].

\section{Representing Clinical Evidence Logic Statements in Established Standards: An Overview}

Steps in the process of translating a unit of evidence into FHIR so that it can be used in CDS are summarized in Figure 1. Recommendations are extracted from evidence sources, including published guidelines, recommendations, systematic reviews, clinical decision rules, and local best practices; each 
extracted recommendation is known as a unit of evidence. Each unit of evidence is then organized into an "If [condition] Then [action]" format which is known as a CELS. Therefore, each
CELS consists of clinical terms and logic operators and has associated metadata (eg, source and author).

Figure 1. Relationship between the standards. HL7: Health Level Seven.

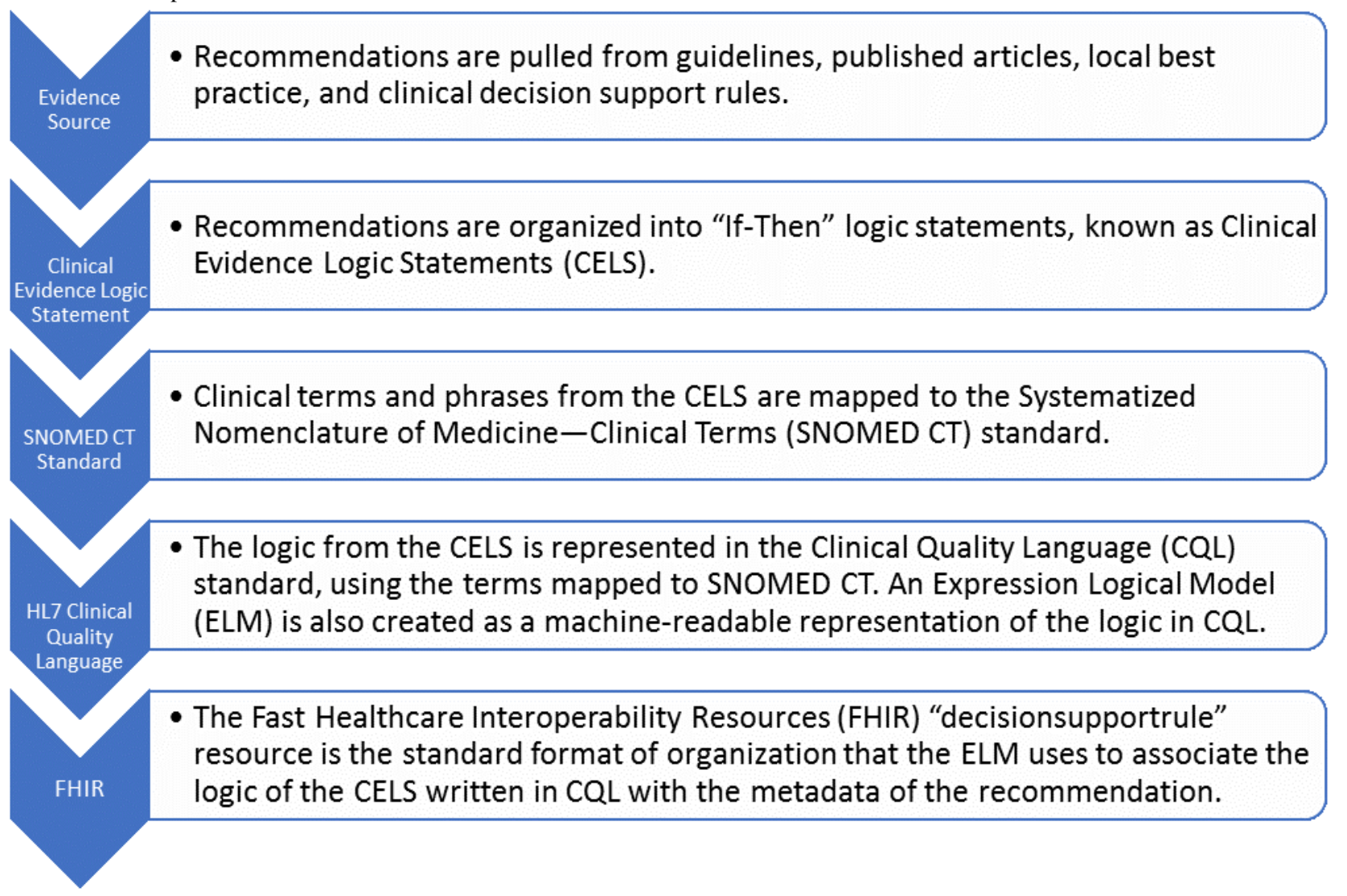

\section{Transforming a Unit of Evidence to Fast Healthcare Interoperability Resources: An Example}

\section{Overview}

An in-depth transformation of a unit of evidence to FHIR is described below using a peer-reviewed article with recommendations for using ventilation-perfusion single-photon emission computed tomography (VQ SPECT) imaging for diagnosing pulmonary embolism [20]. The article recommends using VQ SPECT in patients with suspected pulmonary embolism (PE), and can be written as the following CELS: "If [Suspected PE] Then [VQ SPECT]."

Previous studies related to the HLE have identified three main types of variations in logic: single-decision statements, branching statements, and score-based statements [16]. The "Suspected PE" recommendation is an example of a single-decision statement.

\section{Representing the Terms Using Systematized Nomenclature of Medicine: Clinical Terms}

We modeled "Suspected PE" in SNOMED CT as follows: code "suspected PE": '417113001'. This is an example of a precoordinated match.

\section{Representing Clinical Logic in Clinical Quality Language}

The CQL file is structured into a series of categories including the following:

1. Library: this is the name of the reference file, which is referenced by the secondary ELM file needed for each clinical decision.

2. Using: this term defines the data model that will be used (eg, QUICK).

3. Code System: this identifies the standardized code system, such as SNOMED CT.

4. Value Set: this identifies the specific codes within the code system that will be referenced in the clinical logic; either extensional or intensional value sets can be used.

5. Context: this can either be patient or population. For clinical logic in the HLE, the context is patient, as most data references the patient.

6. Define: this is a statement that creates a local name for conditions (eg, in an "If" statement). 
Figure 2. Suspected pulmonary embolism Clinical Quality Language (CQL) file. QUICK: Quality Information and Clinical Knowledge; SNOMED: Systematized Nomenclature of Medicine; PE: pulmonary embolism.

\author{
USING QUICK \\ codesystem "SNOMED": 'https://www.snomed.org/' \\ code "suspected PE": '417113001' from "SNOMED" display 'suspected PE' \\ context Patient \\ define "C1": \\ exists ["Condition": "suspected PE"]
}

Although one can define an infinite number of subsets, these define statements should be organized and succinct. Naming the define statements creates a local name for all the conditions and rules that either exist or do not exist to make up a defined statement subset; this also allows one to reference the list of conditions in a future define statement, so that the define statements can be stacked. In most cases, the last define statement in the CQL file will be a define statement that contains all the conditions in the "If" statement that must be true to initiate the "Then" portion of the recommendation. A complete CQL file is shown in Figure 2.

\section{Representation in Fast Healthcare Interoperability Resources}

The ELM file (see Figure 3) is the second file necessary to share clinical logic written in CQL. As mentioned previously, the ELM is a machine-readable, canonical representation of the CQL logic, which is the intermediate step in implementing the logic written in CQL. This is where the Event, Context, and Actions are defined. The organization of the ELM file is dictated by the FHIR standard "decisionsupportrule" resource. It can be formatted in the XML, JSON, or Turtle formats; HLE uses XML.

The setup of the ELM file is shown in Textbox 1. Each individual decision rule, which thereby contains an individual action, has its own XML file.

Figure 3. Suspected pulmonary embolism Extensible Markup Language (XML) file. HL7: Health Level Seven; FHIR: Fast Healthcare Interoperability Resources; CELS: Clinical Evidence Logic Statement; VQ SPECT: ventilation-perfusion single-photon emission computed tomography; PE: pulmonary embolism; SNOMED: Systematized Nomenclature of Medicine.

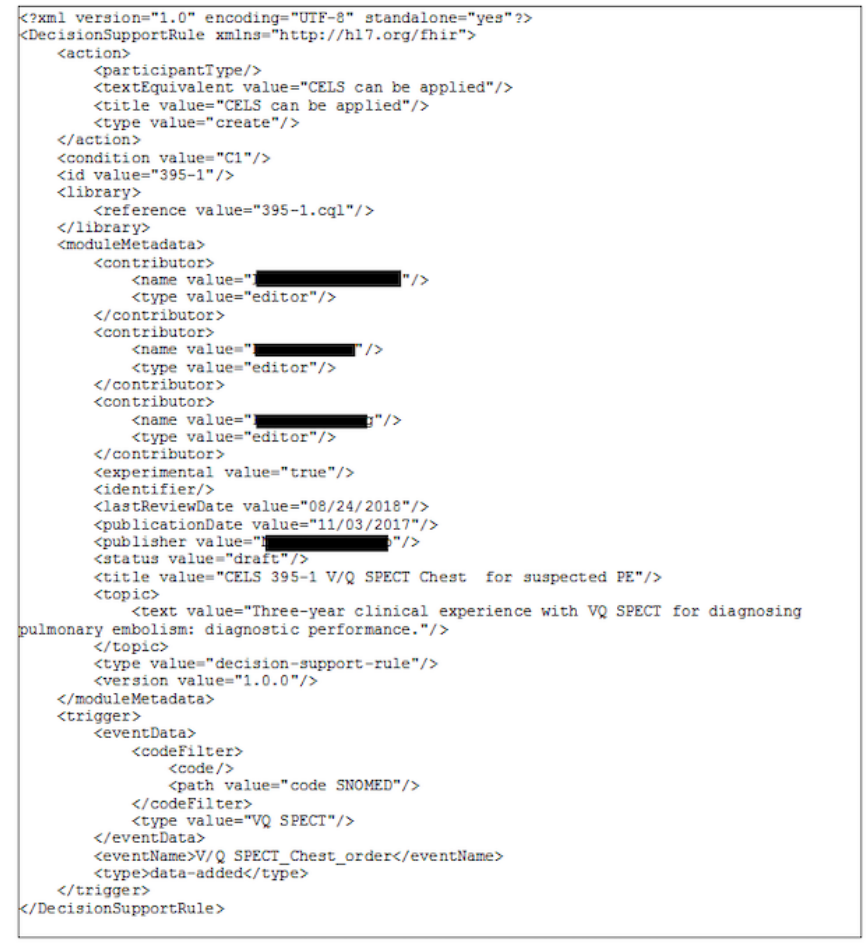


Textbox 1. Setup of the Expression Logical Model (ELM) file.

<action></action>: This portion of the Extensible Markup Language (XML) file references whether the clinical logic deems the test inappropriate or appropriate. The action can either be "Rule can be applied" if the test is appropriate or "Rule cannot be applied" if the test is not appropriate.

$<$ condition value $=/>$ : In this part of the file, the name of the final define statement that contains all the conditions that renders the action true is referenced. Thus, a subset of clinical logic is referenced.

$<$ moduleMetadata $><$ /moduleMetadata $>$ : General information about the library file is referenced (eg, author names and title).

$<$ library $></$ library $>$ : This portion of the XML file references the name of the Clinical Quality Language (CQL) file that contains the logic relevant to the action and condition.

<trigger $></$ trigger $>$ : This portion of the XML file references the clinical order that is related to the clinical logic and contains the event that triggers a decision rule. This trigger is defined in the implementation environment, and not defined in CQL.

The FHIR framework allows for the combination of multiple CQL files with their corresponding XML files. FHIR supports single-decision statements, branching-decision statements, and score-based statements.

\section{Representation of Branching Statements}

Branching statements are recommendations that are applicable to patients with similar indications but fulfil various criteria; for example, recommendations for managing pulmonary embolism in pregnant patients versus nonpregnant patients. Thus, there are more than two CELS generated for the evidence source. The first step of translating these units of evidence is creating a decision tree. Each end point of the decision tree corresponds to a CELS, to be represented in CQL.

\section{Representation of Score-Based Statements}

Score-based units of evidence also produce more than two CELS, corresponding to evidence-based scores; for example, recommendation for managing acute appendicitis for an Acute Inflammatory Response (AIR) score of 5. The ELM files created for each CQL file in the branched and score-based statement follow the same format as the ELM files in the single-decision statement.

\section{Assessing Representation of Clinical Evidence Logic Statements in Established Standards}

The HLE contained a total of 2616 CELS at the time of data analysis for this publication. Among these, we counted the number of CELS that we were able to represent in SNOMED CT, CQL, and FHIR and reported this as a percentage of the total number of cells. For each of these, we characterized those CELS that could not be represented in SNOMED CT and those that could not be represented in CQL. CELS were defined as represented in SNOMED CT when all terms in the CELS could be represented using SNOMED CT. CELS are defined as represented in CQL when the action of the CELS, after the "Then" portion, could be represented in the ELM in the FHIR format.

\section{Results}

We were able to represent terms using SNOMED CT in the temporal component for action ("Then") statements in CQL and FHIR in 755 of 765 (98.7\%) of CELS. Of the completely graded 765 CELS in the evidence library, $17.9 \%(n=137)$ were fully represented using SNOMED CT, CQL, and FHIR (see Table 1).

Reasons why CELS were not adequately represented are included in Textboxes 2-4 and are summarized as follows:

1. Clinical terms are unrepresented using SNOMED CT. Some clinical terms within logic statements contained one or more clinical terms not represented in SNOMED CT (eg, AIR score for acute appendicitis) [21-31].

2. Standard English phrases were unrepresented using SNOMED CT. Some common phrases that were not represented using SNOMED CT include "new feature" or "vehicle rollover."

3. Temporal phrases were unrepresented in CQL. An additional number of CELS were not adequately represented as the "Then" portion of the logic statement because a temporal component could not be represented in CQL (eg, computed tomography [CT] chest in 12 months) and, subsequently, with the FHIR "decisionsupportrule" resource (see Textbox 4).

Table 1. Partially represented CELS ${ }^{\mathrm{a}}$ in the Harvard Medical School Library of Evidence.

\begin{tabular}{ll}
\hline Type of CELS & Number of CELS (N=765), n (\%) \\
\hline CELS fully represented using SNOMED CT ${ }^{\mathrm{b}}, \mathrm{CQL}^{\mathrm{c}}$, and FHIR \\
CELS partially represented using SNOMED CT & $137(17.9)$ \\
CELS partially represented due to CQL & $628(82.1)$ \\
\hline
\end{tabular}

${ }^{\mathrm{a} C E L S}$ : Clinical Evidence Logic Statement.

${ }^{\mathrm{b}}$ SNOMED CT: Systematized Nomenclature of Medicine-Clinical Terms.

${ }^{\mathrm{c} C Q L}$ : Clinical Quality Language.

${ }^{\mathrm{d}}$ FHIR: Fast Healthcare Interoperability Resources. 
Textbox 2. Clinical terms unrepresented using Systematized Nomenclature of Medicine-Clinical Terms (SNOMED CT).

- Acute Inflammatory Response (AIR) score [21]

- $\quad$ Alvarado score [22]

- Canadian Computed Tomography (CT) Head Rule [23]

- $\quad$ Canadian Cervical Spine Rule (CCSR) [24]

- New Orleans/Charity head trauma rule [25]

- $\quad$ National Emergency X-Radiography Utilization Study (NEXUS) head trauma rule [26]

- Magnetic resonance imaging (MRI) shoulder with dedicated metal suppression protocol

- $\mathrm{O}_{2}$ saturation on room air

- $\quad$ Optimizing imaging in suspected appendicitis (OPTIMAP) score [27]

- $\quad$ Revised Geneva (rGeneva) score [28]

- Simple calculated osteoporosis risk estimation (SCORE) score [29]

- $\quad$ Simplified Motor Score (SMS) [30]

- Sex, timing, origin, nausea, erythrocytes (STONE) score [31]

Textbox 3. Standard English phrases unrepresented using Systematized Nomenclature of Medicine—Clinical Terms (SNOMED CT).

- $\quad$ New feature

- Suitable candidate

- Time-of-flight (TOF) magnetic resonance angiography (MRA)

- Vehicle rollover

Textbox 4. Temporal phrases unrepresented in Clinical Quality Language (CQL).

- Computed tomography (CT) chest in 12 months

- $\quad$ CT chest in 18-24 months

- $\quad$ CT chest in 3 months, 9 months, And 24 months

- $\mathrm{CT}$ chest in 3-6 months

- $\quad$ CT chest in 6-12 months

- $\quad$ T chest in 9-12 months And 24 months

- $\quad$ Low-dose CT annually for 3 years

Examples of partially unrepresented CELS include:

1. Alvarado score for suspected appendicitis; this is an example of an evidence source with three partially represented CELS, since the term "Alvarado score" does not exist in the SNOMED CT standard ontology, as indicated by the asterisk:

a. If [Alvarado score* >=4] And [Alvarado score* <=6] Then [CT abdomen]

b. If [Alvarado score* <4] Then Not [CT Abdomen]

c. If [Alvarado score* $>6$ ] Then Not [CT Abdomen]

2. Guidelines for management of small pulmonary nodules detected on CT scans-a statement from the Fleischner Society [32]:

a. If [pulmonary nodule on chest CT] And [nodule size $<=4 \mathrm{~mm}$ ] And [high risk] Then [CT chest in 12 months] b. If [pulmonary nodule on chest CT] And [nodule size $>4 \mathrm{~mm}$ ] And [nodule size <=6] And [low risk] Then [CT Chest in 12 months]

c. If [pulmonary nodule on chest CT] And [nodule size $>4 \mathrm{~mm}$ ] And [nodule size <=6] And [high risk] Then [CT chest in 6-12 months]

These CELS are examples of partially represented CELS due to actions such as "CT chest in 6-12 months" and "CT chest in 12 months." These actions contain a future temporal component that cannot be represented in CQL. CT chest can be represented in a define statement. However, a define statement in CQL for scheduling a procedure at a future time cannot be created. Furthermore, value sets for terms such as "high risk" are not available. 


\section{Discussion}

\section{Principal Findings}

Overall, $17.9 \%(137 / 765)$ of CELS were represented as shareable CDS knowledge artifacts using existing standards, SNOMED CT, FHIR, and CQL to promote and accelerate adoption of evidence-based practice. More work to represent imaging-related CELS need to be undertaken to standardize clinical knowledge included in the HLE. A few limitations to utilizing these standards for CDS implementation in the evidence library were identified. While SNOMED CT is robust, some terms do not exist in its ontology. For example, names for known rules or scores such as "Revised Geneva score" [28] cannot be represented. The HLE is currently in the process of creating an add-on set of terms in SNOMED CT so that these terms will have an ID and mapping.

In addition, English words, which contribute to the meaning of a clinical recommendation (eg, "high risk" and "suitable candidate"), may not be represented using SNOMED CT. In those situations, one can substitute terms that are in SNOMED CT that are a synonym or close in meaning to the original term. Use of value sets to enumerate concepts that may map to a criterion in a recommendation may be useful. However, these mappings are not always exact and may change the interpretation of the clinical recommendation. This limitation can also possibly be amended through an add-on set of terms to SNOMED CT. SNOMED CT is updated twice yearly and updates can include newly added concepts. In addition, developing an add-on set of terms can be expedited by creating more value sets, specifically sets of code from hierarchy-based definitions that are algorithmically defined (ie, intensional value sets) or enumerated (ie, extensional value sets). These can be disseminated publicly (eg, via the Value Set Authority Center) to accelerate cross-organizational efforts for terminology standardization. More importantly, guidelines and recommendations should be

\section{Acknowledgments}

The authors would like to thank Ms Laura Peterson for reviewing the manuscript.

\section{Authors' Contributions}

All authors contributed to the study design and data acquisition; EO, RL, and RK are responsible for data analysis and interpretation. All authors contributed significant intellectual content during the manuscript preparation and revisions, approved the final version, and accept accountability for the overall integrity of the research process and the manuscript.

\section{Conflicts of Interest}

None declared.

\section{References}

1. Khorasani R, Hentel K, Darer J, Langlotz C, Ip I, Manaker S, et al. Ten commandments for effective clinical decision support for imaging: Enabling evidence-based practice to improve quality and reduce waste. AJR Am J Roentgenol 2014 Nov;203(5):945-951. [doi: 10.2214/AJR.14.13134] [Medline: 25341131]

2. Department of Health and Human Services. Federal Register (volume 72, issue 209). 2009 Oct 30. HIPAA administrative simplification: Enforcement URL: https://www.govinfo.gov/content/pkg/PLAW-111publ5/pdf/PLAW-111publ5.pdf [accessed 2019-05-03] [WebCite Cache ID 787zG6aj2] 
3. Gold M, McLaughlin C. Assessing HITECH implementation and lessons: 5 years later. Milbank Q 2016 Sep;94(3):654-687 [FREE Full text] [doi: 10.1111/1468-0009.12214] [Medline: 27620687]

4. Medicare: Considerations for Expansion of the Appropriate Use Criteria Program. Washington, DC: US Government Accountability Office; 2015 Sep 30. URL: https://www.gao.gov/assets/680/672856.pdf [accessed 2019-05-04] [WebCite Cache ID 7897g4drI]

5. Bright T, Wong A, Dhurjati R, Bristow E, Bastian L, Coeytaux R, et al. Effect of clinical decision-support systems: A systematic review. Ann Intern Med 2012 Jul 03;157(1):29-43. [doi: 10.7326/0003-4819-157-1-201207030-00450] [Medline: $\underline{22751758]}$

6. Dunne R, Ip I, Abbett S, Gershanik E, Raja A, Hunsaker A, et al. Effect of evidence-based clinical decision support on the use and yield of CT pulmonary angiographic imaging in hospitalized patients. Radiology 2015 Jul;276(1):167-174 [FREE Full text] [doi: 10.1148/radiol.15141208] [Medline: 25686367]

7. Semelka R, Armao D, Elias JJ, Huda W. Imaging strategies to reduce the risk of radiation in CT studies, including selective substitution with MRI. J Magn Reson Imaging 2007 May;25(5):900-909. [doi: 10.1002/jmri.20895] [Medline: 17457809]

8. US National Library of Medicine. Bethesda, MD: US National Library of Medicine, National Institutes of Health, Health \& Human Services; 2009. SNOMED CT URL: http://www.nlm.nih.gov/research/umls/Snomed/snomed main.html [accessed 2019-04-21] [WebCite Cache ID 77pK7mQhp]

9. Lin Y, Staes C, Shields D, Kandula V, Welch B, Kawamoto K. Design, development, and initial evaluation of a terminology for clinical decision support and electronic clinical quality measurement. AMIA Annu Symp Proc 2015;2015:843-851 [FREE Full text] [Medline: 26958220]

10. Benson T, Grieve G. Principles of Health Interoperability: SNOMED CT, HL7 and FHIR. 3rd edition. London, UK: Springer-Verlag; 2016.

11. SNOMED CT: Compositional Grammar v2.3.1 - Specification and Guide. London, UK: International Health Terminology Standards Development Organisation (IHTSDO); 2016 Nov 11. URL: https://tinyurl.com/y238f5sk [accessed 2019-05-04] [WebCite Cache ID 789B9FqZx]

12. HL7.org. 2018. HL7 Standard: Clinical Quality Language Specification, Release 1 STU3 (CQL 1.3) URL: https://cql. hl7.org/ [accessed 2019-04-21] [WebCite Cache ID 77pLYH8Db]

13. HL7. HL7.org. 2019. Quick data model URL: http://hl7.org/fhir/us/qicore/2018Jan/quick/index.html [accessed 2019-04-21] [WebCite Cache ID 77pM9tCpe]

14. HL7.org. FHIR STU 3 Candidate URL: http://hl7.org/fhir/2016May/resourcelist.html [accessed 2019-04-21] [WebCite Cache ID 77pMLBD3Z]

15. Lacson R, Raja A, Osterbur D, Ip I, Schneider L, Bain P, et al. Assessing strength of evidence of appropriate use criteria for diagnostic imaging examinations. J Am Med Inform Assoc 2016 Jan;23(3):649-653. [doi: 10.1093/jamia/ocv194] [Medline: 26911819]

16. Varada S, Lacson R, Raja A, Ip I, Schneider L, Osterbur D, et al. Characteristics of knowledge content in a curated online evidence library. J Am Med Inform Assoc 2018 May 01;25(5):507-514. [doi: 10.1093/jamia/ocx092] [Medline: 29092054]

17. Harvard Medical School Library of Evidence. URL: http://libraryofevidence.med.harvard.edu/ [accessed 2019-04-21] [WebCite Cache ID 77pMU1Cv7]

18. Choosing Wisely. URL: http://www.choosingwisely.org/ [accessed 2019-04-21] [WebCite Cache ID 77pMei8Hj]

19. Centers for Medicare \& Medicaid Services (CMS), HHS. Medicare program: Medicare clinical diagnostic laboratory tests payment system. Final rule. Fed Regist 2016 Jun 23;81(121):41035-41101 [FREE Full text] [Medline: 27373013]

20. Grüning T, Drake B, Farrell S, Nokes T. Three-year clinical experience with VQ SPECT for diagnosing pulmonary embolism: Diagnostic performance. Clin Imaging 2014;38(6):831-835. [doi: 10.1016/j.clinimag.2014.04.003] [Medline: 24852679]

21. Scott A, Mason S, Arunakirinathan M, Reissis Y, Kinross J, Smith J. Risk stratification by the Appendicitis Inflammatory Response score to guide decision-making in patients with suspected appendicitis. Br J Surg 2015 Apr;102(5):563-572. [doi: 10.1002/bjs.9773] [Medline: 25727811]

22. McKay R, Shepherd J. The use of the clinical scoring system by Alvarado in the decision to perform computed tomography for acute appendicitis in the ED. Am J Emerg Med 2007 Jun;25(5):489-493. [doi: 10.1016/j.ajem.2006.08.020] [Medline: $\underline{17543650]}$

23. Stiell I, Wells G, Vandemheen K, Clement C, Lesiuk H, Laupacis A, et al. The Canadian CT Head Rule for patients with minor head injury. Lancet 2001 May 05;357(9266):1391-1396. [Medline: 11356436]

24. Coffey F, Hewitt S, Stiell I, Howarth N, Miller P, Clement C, et al. Validation of the Canadian c-spine rule in the UK emergency department setting. Emerg Med J 2011 Oct;28(10):873-876. [doi: 10.1136/emj.2009.089508] [Medline: 21068171]

25. Haydel M, Preston C, Mills T, Luber S, Blaudeau E, DeBlieux P. Indications for computed tomography in patients with minor head injury. N Engl J Med 2000 Jul 13;343(2):100-105. [doi: 10.1056/NEJM200007133430204] [Medline: 10891517]

26. Mower W, Hoffman J, Herbert M, Wolfson A, Pollack CJ, Zucker M, NEXUS II Investigators. Developing a decision instrument to guide computed tomographic imaging of blunt head injury patients. J Trauma 2005 Oct;59(4):954-959. [Medline: 16374287] 
27. Leeuwenburgh M, Stockmann H, Bouma W, Houdijk A, Verhagen M, Vrouenraets B, OPTIMAP Study Group. A simple clinical decision rule to rule out appendicitis in patients with nondiagnostic ultrasound results. Acad Emerg Med 2014 May;21(5):488-496 [FREE Full text] [doi: 10.1111/acem.12374] [Medline: 24842498]

28. Le Gal G, Righini M, Roy P, Sanchez O, Aujesky D, Bounameaux H, et al. Prediction of pulmonary embolism in the emergency department: The revised Geneva score. Ann Intern Med 2006 Feb 07;144(3):165-171. [Medline: 16461960]

29. Cadarette S, Jaglal S, Murray T. Validation of the simple calculated osteoporosis risk estimation (SCORE) for patient selection for bone densitometry. Osteoporos Int 1999;10(1):85-90. [doi: 10.1007/s001980050199] [Medline: 10501785]

30. Haukoos J, Gill M, Rabon R, Gravitz C, Green S. Validation of the Simplified Motor Score for the prediction of brain injury outcomes after trauma. Ann Emerg Med 2007 Jul;50(1):18-24. [doi: 10.1016/j.annemergmed.2006.10.004] [Medline: 17113193]

31. Moore C, Bomann S, Daniels B, Luty S, Molinaro A, Singh D, et al. Derivation and validation of a clinical prediction rule for uncomplicated ureteral stone--the STONE score: Retrospective and prospective observational cohort studies. BMJ 2014 Mar 26;348:g2191 [FREE Full text] [doi: 10.1136/bmj.g2191] [Medline: 24671981]

32. MacMahon H, Austin J, Gamsu G, Herold C, Jett J, Naidich D, Fleischner Society. Guidelines for management of small pulmonary nodules detected on CT scans: A statement from the Fleischner Society. Radiology 2005 Nov;237(2):395-400. [doi: 10.1148/radiol.2372041887] [Medline: 16244247]

\section{Abbreviations}

AIR: Acute Inflammatory Response

CCSR: Canadian Cervical Spine Rule

CDS: clinical decision support

CELS: Clinical Evidence Logic Statement

CQL: Clinical Quality Language

CT: computed tomography

ELM: Expression Logical Model

FHIR: Fast Healthcare Interoperability Resources

HL7: Health Level Seven

HLE: Harvard Medical School Library of Evidence

IT: information technology

JSON: JavaScript Object Notation

MRA: magnetic resonance angiography

MRI: magnetic resonance imaging

NEXUS: National Emergency X-Radiography Utilization Study

OPTIMAP: optimizing imaging in suspected appendicitis

PAMA: Protecting Access to Medicare Act

PE: pulmonary embolism

QUICK: Quality Information and Clinical Knowledge

rGeneva: Revised Geneva

SCORE: simple calculated osteoporosis risk estimation

SMS: Simplified Motor Score

SNOMED CT: Systematized Nomenclature of Medicine-Clinical Terms

STONE: sex, timing, origin, nausea, erythrocytes

TOF: time-of-flight

VQ SPECT: ventilation-perfusion single-photon emission computed tomography

XML: Extensible Markup Language

Edited by G Eysenbach; submitted 01.02.19; peer-reviewed by J Durack, D Willett; comments to author 13.03.19; revised version
received 21.03.19; accepted 24.03.19; published 13.05.19
Please cite as:
Odigie E, Lacson R, Raja A, Osterbur D, Ip I, Schneider L, Khorasani R
Fast Healthcare Interoperability Resources, Clinical Quality Language, and Systematized Nomenclature of Medicine_Clinical Terms
in Representing Clinical Evidence Logic Statements for the Use of Imaging Procedures: Descriptive Study
JMIR Med Inform 2019;7(2):e13590
URL: http://medinform.jmir.org/2019/2/e13590/
doi: $10.2196 / 13590$
PMID: $\underline{31094359}$


CEseosa Odigie, Ronilda Lacson, Ali Raja, David Osterbur, Ivan Ip, Louise Schneider, Ramin Khorasani. Originally published in JMIR Medical Informatics (http://medinform.jmir.org), 13.05.2019. This is an open-access article distributed under the terms of the Creative Commons Attribution License (https://creativecommons.org/licenses/by/4.0/), which permits unrestricted use, distribution, and reproduction in any medium, provided the original work, first published in JMIR Medical Informatics, is properly cited. The complete bibliographic information, a link to the original publication on http://medinform.jmir.org/, as well as this copyright and license information must be included. 